\title{
Treatment response to osimertinib in a patient with leptomeningeal metastasis from lung adenocarcinoma following failure of gefitinib and erlotinib: A case report
}

\author{
JUNJUN LI, XIAOMEI LIU and CAIJUN YUAN \\ Department of Oncology, The First Affiliated Hospital of Jinzhou Medical University, Jinzhou, Liaoning 121000, P.R. China
}

Received March 27, 2018; Accepted June 6, 2018

DOI: $10.3892 / \mathrm{mco} .2018 .1666$

\begin{abstract}
Epidermal growth factor receptor-tyrosine kinase inhibitors (EGFR-TKIs) are effective in the treatment of advanced non-small-cell lung cancer (NSCLC) with leptomeningeal metastases (LM); however, a proportion of the patients with resistant tumors do not benefit from EGFR-TKI treatment. In the present study the case of a female patient with advanced lung adenocarcinoma harboring the EGFR L858R mutation (encoded in exon 21) who developed intracranial metastases following treatment with erlotinib after gefitinib failure is reported. The patient achieved a partial response (PR) after four cycles of chemotherapy with pemetrexed/cisplatin. However, after 4 months, LM were detected. The patient was treated with osimertinib, a third-generation EGFR-TKI, but the LM continued to progress and the patient eventually succumbed to the disease (overall survival, 6 months). Therefore, LM in patients without the EGFR T790M mutation (encoded in exon 20) appear to be resistant to treatment with the third-generation TKI osimertinib, which may be associated with human epidermal growth factor receptor 2 amplification. Further clinical trials are required to confirm our results.
\end{abstract}

\section{Introduction}

Lung cancer is the leading cause of cancer-related mortality and NSCLC is responsible for $\sim 85 \%$ of lung cancer cases worldwide (1). Advanced NSCLC is associated with a higher risk of leptomeningeal metastasis (LM) compared with other tumors. Due to the limitations of the blood-brain barrier, traditional cytotoxic drugs are not effective, and patients with brain metastases form NSCLC have a poor prognosis, as their

Correspondence to: Professor Caijun Yuan, Department of Oncology, The First Affiliated Hospital of Jinzhou Medical University, 2 The Fifth Section of Renmin Street, Guta, Jinzhou, Liaoning 121000, P.R. China

E-mail: 2151208698@qq.com

Key words: lung adenocarcinoma, leptomeningeal metastases, osimertinib, epidermal growth factor receptor-tyrosine kinase inhibitors, human epidermal growth factor receptor 2 amplification condition deteriorates rapidly due to the lack of standard and effective treatments. Radiotherapy of LM requires a large irradiation field, is associated with severe adverse reactions, and generally has a poor prognosis. Previous studies have demonstrated that epidermal growth factor receptor-tyrosine kinase inhibitors (EGFR-TKIs) can improve the prognosis of these patients $(2,3)$. However, EGFR mutation-positive patients eventually develop resistance to EGFR-TKIs, the most frequent reason being a secondary EGFR T790M mutation encoded in exon 20 (4).

Osimertinib, a third-generation EGFR-TKI, was recently approved for use in NSCLC patients who have been previously treated with EGFR-TKIs and/or chemotherapy and who harbor the EGFR T790M mutation (5,6). Early results from a phase I study (BLOOM) indicate that osimertinib is effective for LM treatment, regardless of whether there is a T790M mutation (7). We herein report the case of a patient with advanced NSCLC with LM who was treated with osimertinib.

\section{Case report}

A 59-year-old woman was admitted to The First Affiliated Hospital of Jinzhou Medical University (Jinzhou, China) in December 2015, following detection of a mass in her left lung on routine physical examination. The patient had never been a smoker and had no other significant medical history. A computed tomography (CT) scan revealed a mass in the left lung and small nodules in the inferior lobe of the right lung. The patient's carcinoembryonic antigen (CEA) level was $422.0 \mathrm{ng} / \mathrm{ml}$ (normal range: $0.0-3.4 \mathrm{ng} / \mathrm{ml}$ ) higher than normal, and it significantly increased to $15854.2 \mathrm{ng} / \mathrm{ml}$ when the patient's condition worsened (Fig. 1). The patient was diagnosed with lung adenocarcinoma by fiberoptic bronchoscopy and biopsy. Mutation analysis of EGFR in adenocarcinoma cells revealed an L858R mutation in the EGFR gene. A bone emission computerized tomography scan revealed metastasis to the thoracic and lumbar vertebrae. The patient was initiated on $250 \mathrm{mg}$ of gefitinib orally once a day in January 2016. After 1 month of treatment with gefitinib, the lung tumor exhibited partial response (PR). In April 2016, the mass in the left lung had increased in size as detected during CT examination, and gefitinib was replaced with erlotinib (150 mg/day). However, the patient developed progressive disease on erlotinib. 
In September 2016, a new metastasis in the brain was detected during brain magnetic resonance imaging (MRI) examination (Fig. 2A). The patient was administered four cycles of pemetrexed/cisplatin, and the cranial tumor exhibited a PR that lasted for 5 months (Fig. 2B). However, in February 2017, the patient developed headache, dizziness and vomiting. An MRI of the patients' spine revealed multiple metastases to the spine (Fig. 3). Based on this finding and taking into consideration the risks of lumbar puncture, the options were discussed with the patient's family, and the family declined both the lumbar puncture and cerebrospinal fluid examination. A brain MRI revealed linear high-signal intensities in parts of the meninges, and linear hardening was observed with enhanced scanning. Thus, LM was diagnosed (Fig. 4A). Despite receiving a series of symptomatic supportive treatments, the patient's symptoms of intracranial metastases were not relieved. It was recommended to the patient's family that the pulmonary lesions were biopsied for pathological detection of gene mutations. The family members considered the patient's current symptoms and refused biopsy, but agreed to genetic testing of a blood sample. In February 2017, The Beijing Genomics Institute was commissioned to perform genetic testing on a blood sample. The EGFR T790M mutation encoded in EGFR exon 20 was not detected in this patient, but human epidermal growth factor receptor 2 (HER2) amplification was detected instead. Treatment with oral osimertinib was initiated at a dose of $80 \mathrm{mg}$ per day in February 2017. A brain MRI revealed that LM had progressed, and the patient's symptoms worsened after 1 month of treatment with osimertinib (Fig. 4B). The dose of the drug was increased to $160 \mathrm{mg}$ per day. However, after 1 month of treatment, LM progressed again (Fig. 4C), and the CEA level also increased from 8613.7 to $11239.9 \mathrm{ng} / \mathrm{ml}$ (normal range: $0.0-3.4 \mathrm{ng} / \mathrm{ml}$ ) during treatment with osimertinib (Fig. 1). The patient's symptoms worsened, and the LM continued to progress. Due to the deterioration of the performance status (PS 4), the patient received supportive care and succumbed to the disease 3 months following the worsening of the meningeal metastasis symptoms.

This case report was approved by the Ethics Committees of Jinzhou Medical University and written informed consent was obtained from the patient regarding the publication of the case details and associated images.

\section{Discussion}

Patients with advanced NSCLC and LM have a poor prognosis. Our patient was a woman with advanced lung adenocarcinoma who had no smoking history. The primary lesions harbored exon-EGFR19 mutations. Based on genetic testing, the patient was sensitive to EGFR-TKI treatment and the extracranial conditions were briefly controlled. Erlotinib was used to replace gefitinib as EGFR-TKI treatment following development of gefitinib resistance and disease progression. However, brain metastases occurred during gefitinib treatment with progression of peripheral lesions. In addition to the standard whole-brain radiotherapy, some studies have reported that EGFR-TKIs may improve the prognosis of NSCLC patients. EGFR mutation is an oncogenic driver mutation, and it has been demonstrated that individual NSCLC patients with oncogenic drivers who receive

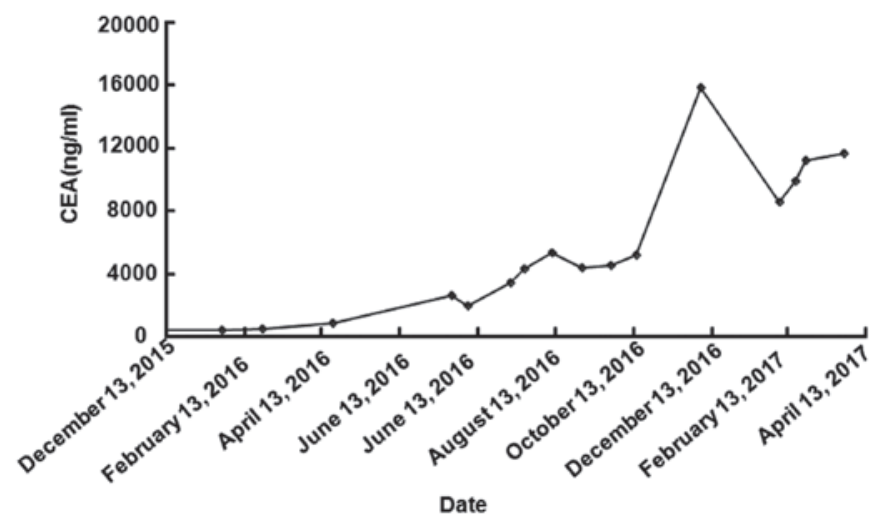

Figure 1. Serum carcinoembryonic antigen (CEA) levels during the course of the whole treatment. The patient's CEA value was higher than normal, and it increased significantly when the patient's condition deteriorated.

a matched targeted agent exhibit improved prognosis, and treatment with an EGFR-TKI is recommended as first-line therapy for EGFR mutation-positive NSCLC patients (8). However, resistance develops rapidly, the main reason for which is a secondary T790M mutation within the ATP site of the receptor $(9,10)$, with a mutation probability of $\sim 60 \%$. A third-generation EGFR-TKI, osimertinib, was recently found to be of clinical value for NSCLC patients who develop a secondary EGFR T790M mutation, which is the most frequent reason for resistance to first-line treatment with EGFR-TKIs (4,5). Recently, the BLOOM trial found osimertinib to be effective in patients with advanced NSCLC who developed LM, regardless of the presence of a T790M mutation (7).

EGFR T790M has been identified as the most common mechanism underlying acquired resistance, while MET amplification, HER2 amplification and small-cell histological transformation occur less frequently. Although EGFR T790M mutation encoded in EGFR exon 20 was not detected in our patient, HER2 and MYC amplification, lack of CDKN2A, TP53 exon 4 and 6, ATP1B1 exon 21, and APC exon 5 mutations were detected. In fact, HER2 amplification may be a more common finding at the time of resistance development (11). The HER2 copy number appeared to be associated with gefitinib sensitivity in EGFR-positive patients treated with gefitinib in North America and Europe (12). The prognostic role of EGFR in lung cancer is not well defined, but previous studies have demonstrated that patients overexpressing EGFR and HER2 have a poor prognosis (13-15). Conversely, current findings suggest that high copy numbers of the HER2 gene increase sensitivity to gefitinib therapy (15). Preclinical data have revealed that tumors overexpressing HER2 are the most sensitive to gefitinib, possibly because this drug induces sequestration of HER 2 and HER 3 receptors in an inactive heterodimer configuration with the EGFR (16). Conflicting results have also been reported regarding the interaction between EGFR mutation and EGFR and HER2 copy numbers $(10,17-19)$.

The efficacy of EGFR-TKIs, such as gefitinib, erlotinib and afatinib, in the treatment of NSCLC has been proven, particularly in EGFR mutation-positive patients; however, resistance develops rapidly. Osimertinib has exhibited good penetration 

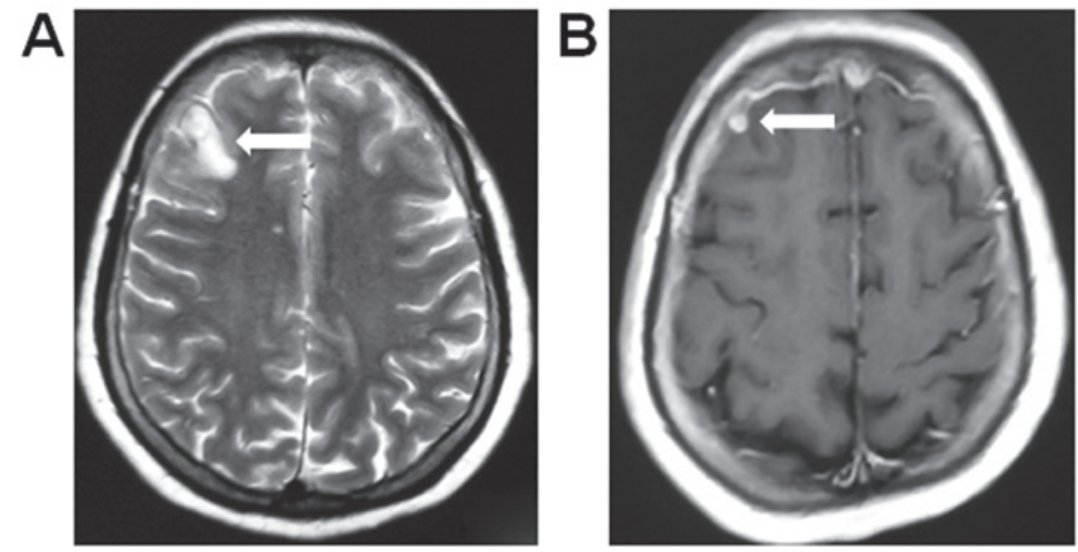

Figure 2. Computed tomography scan showing the intracranial metastases. (A) Prior to treatment (September 19, 2016). (B) After treatment with four cycles of chemotherapy with pemetrexed/cisplatin (December 15, 2016), the intracranial metastases (arrows) appeared smaller compared with 3 months earlier.

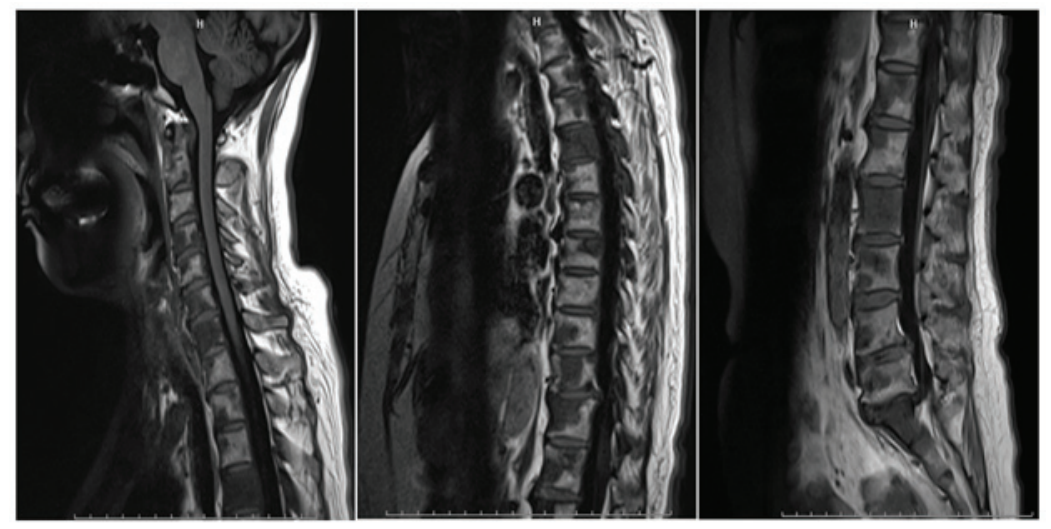

Figure 3. Brain magnetic resonance imaging (February 6, 2017) showing multiple bone lesions with osseous destruction and signal abnormalities in the vertebrae and appendages, indicating multiple metastases to the spine.
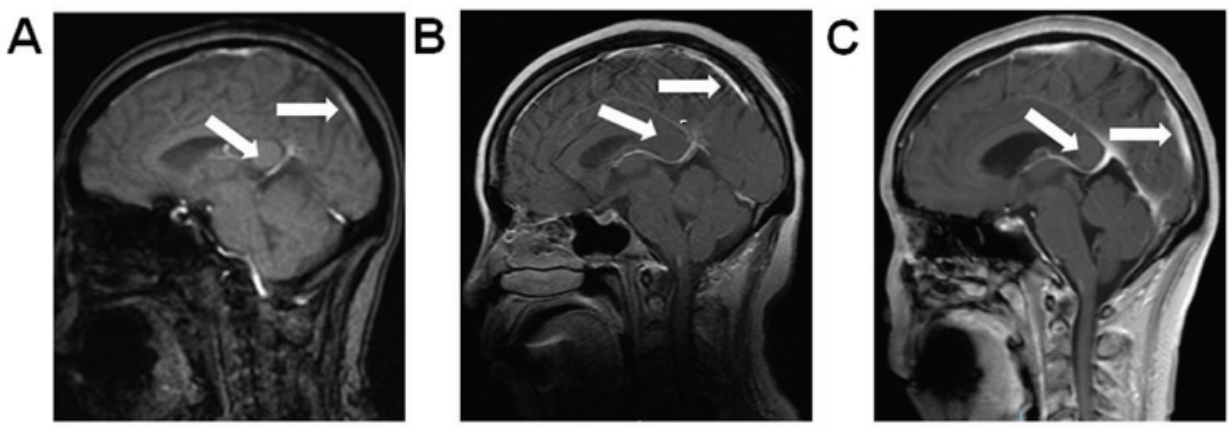

Figure 4. Brain magnetic resonance imaging showing the leptomeningeal metastasis (LM). (A) Prior to treatment (February 6, 2017). (B) After 1 month of treatment with osimertinib at a dose of $80 \mathrm{mg}$ per day (April 22, 2017), LM had progressed and the patient's symptoms worsened. (C) After 1 month of treatment with osimertinib at a dose of $160 \mathrm{mg}$ per day (May 27, 2017), the patient's symptoms worsened and the LM progressed further. Arrows indicate the LM.

through the blood-brain barrier, delaying the development of leptomeningeal carcinomatosis in EGFR mutation-positive cases.

Unfortunately, our patient, who had lung adenocarcinoma resistant to gefitinib and erlotinib treatment and was negative for the EGFR T790M mutation, was not sensitive to osimertinib. HER2 amplification may be the mechanism underlying acquired resistance. Previous studies have reported that patients overexpressing EGFR and HER2 have a poor prognosis. Conversely, current findings suggest that high copy numbers of the HER2 gene increase sensitivity to gefitinib therapy. Further studies are required to elucidate this association and the role of HER 2 protein status among patients treated with reversible EGFR-TKIs.

\section{Acknowledgements}

Not applicable. 


\section{Funding}

This study was supported by the Liaoning Province Science and Technology Project (grant no. 2010010280-401).

\section{Availability of data and materials}

Not applicable.

\section{Authors' contributions}

JL collected the data and drafted the manuscript; XL and CY analyzed and interpreted the patient data regarding osimertinib resistance. JL, XL and CY were involved in revising the manuscript, All the authors have read and approved the final version of the manuscript.

\section{Ethics approval and consent to participate}

This case report was approved by the Ethics Committees of Jinzhou Medical University.

\section{Consent for publication}

Written informed consent was obtained from the patient regarding the publication of the case details and associated images.

\section{Competing interests}

The authors declare that they have no competing interests to disclose.

\section{References}

1. Jemal A, Siegel R, Xu J and Ward E: Cancer statistics, 2010. CA Cancer J Clin 60: 277-300, 2010.

2. Jorge SE, Kobayashi SS and Costa DB: Epidermal growth factor receptor(EGFR) mutations in lung cancer: Preclinical and clinical data. Braz J Med Biol Res 47: 929-939, 2014.

3. Yi HG, Kim HJ, Kim YJ, Han SW, Oh DY, Lee SH, Kim DW, Im SA, Kim TY, Kim CS, et al: Epidermal growth factor receptor (EGFR) tyrosine kinase inhibitors (TKIs) are effective for leptomeningeal metastasis from non-small cell lung cancer patients with sensitive EGFR mutation or other predictive factors of good response for EGFR TKI. Lung Cancer 65: 80-84, 2009.

4. Yu HA, Arcila ME, Rekhtman N, Sima CS, Zakowski MF, Pao W, Kris MG, Miller VA, Ladanyi M and Riely GJ: Analysis of tumor specimens at the time of acquired resistance to EGFR-TKI therapy in 155 patients with EGFR-mutant lung cancers. Clin Cancer Res 19: 2240-2247, 2013.

5. Jänne PA, Yang JC, Kim DW, Planchard D, Ohe Y, Ramalingam SS, Ahn MJ, Kim SW, Su WC, Horn L, et al: AZD9291 in EGFR inhibitor-resistant non-small-cell lung cancer. N Engl J Med 372: 1689-1699, 2015.

6. Uemura T, Oguri T, Okayama M, Furuta H, Kanemitsu Y, Takakuwa O, Ohkubo H, Takemura M, Maeno K, Ito Y and Niimi A: Dramatic intracranial response to osimertinib in a poor performance status patient with lung adenocarcinoma harboring the epidermal growth factor receptor T790M mutation: A case report. Mol Clin Oncol 6: 525-528, 2017.
7. Wang J, Kim DS and Kim SW: Osimertinib activity in patients (pts) with leptomeningeal (LM) disease from non-small cell lung cancer (NSCLC): Updated results from BLOOM, a phase I study. ASCO Meeting Abs 34, 2016.

8. Grigoriu B, Berghmans T and Meert AP: Management of EGFR mutated nonsmall cell lung carcinoma patients. Eur Respir J 45: 1132-1141, 2015.

9. Gainor JF and Shaw AT: Emerging paradigms in the development of resistance to tyrosine kinase inhibitors in lung cancer. J Clin Oncol 31: 3987-3996, 2013.

10. Chong CR and Jänne PA: The quest to overcome resistance to EGFR-targeted therapies in cancer. Nat Med 19: 1389-1400, 2013.

11. Yu HA, Arcila ME, Rekhtman N, Sima CS, Zakowski MF, Pao W, Kris MG, Miller VA, Ladanyi M and Riely GJ: Analysis of tumor specimens at the time of acquired resistance to EGFR TKI therapy in 155 patients with EGFR-mutant lung cancers. Clin Cancer Res 19: 2240-2247, 2013.

12. Cappuzzo F, Varella-Garcia M, Shigematsu H, Domenichini I, Bartolini S, Ceresoli GL, Rossi E, Ludovini V, Gregore V, Toschi L, et al: Increased HER2 gene copy number is associated with response to gefitinib therapy in epidermal growth factor receptor-positive non-small-cell lung cancer patients. J Clin Oncol 23: 5007-5018, 2005.

13. Kim EK, Kim KA, Lee CY and Shim HS: The frequency and clinical impact of HER2 alterations in lung adenocarcinoma. PLoS One 12: e0171280, 2017.

14. Hirsch FR, Varella-Garcia M, Bunn PA Jr, Di Maria MV, Veve R, Bremmes RM, Barón AE, Zeng C and Franklin WA: Epidermal growth factor receptor in non-small-cell lung carcinomas: Correlation between gene copy number and protein expression and impact on prognosis. J Clin Oncol 21: 3798-3807, 2003.

15. Tan D, Deeb G, Wang J, Slocum HK, Winston J, Wiseman S, Beck A, Sait S, Anderson T, Nwogu C, et al: HER-2/neu protein expression and gene alteration in stage I-IIIA non-small-cell lung cancer: A study of 140 cases using a combination of high throughput tissue microarray, immunohistochemistry, and fluorescent in situ hybridization. Diagn Mol Pathol 12: 201-211, 2003.

16. Cappuzzo F, Varella-Garcia M, Shigematsu H, Domenichini I, Bartolini S, Ceresoli GL, Rossi E, Ludovini V, Gregorc V, Toschi L, et al: Increased HER2 gene copy number is associated with response to gefitinib therapy in epidermal growth factor receptor-positive non-small-cell lung cancer patients. J Clin Oncol 23: 5007-5018, 2005.

17. Cappuzzo F, Hirsch FR, Rossi E, Bartolini S, Ceresoli GL, Bemis L, Haney J, Witta S, Danenberg K, Domenichini I, et al: Epidermal growth factor receptor gene and protein and gefitinib sensitivity in non-small-cell lung cancer. J Natl Cancer Inst 97: 643-655, 2005.

18. Takano T, Ohe Y, Sakamoto H, Tsuta K, Matsuno Y, Tateishi U, Yamamoto S, Nokihara H, Yamamoto N, Sekine I, et al: Epidermal growth factor receptor gene mutations and increased copy numbers predict gefitinib sensitivity in patients with recurrent non-small-cell lung cancer. J Clin Oncol 23: 6829-6837, 2005.

19. Bell DW, Lynch TJ, Haserlat SM, Harris PL, Okimoto RA, Brannigan BW, Sgroi DC, Muir B, Riemenschneider MJ, Iacona RB, et al: Epidermal growth factor receptor mutations and gene amplification in non-small-cell lung cancer: Molecular analysis of the IDEAL/INTACT gefitinib trials. J Clin Oncol 23: 8081-8092, 2005. 